\title{
Multiplicative Multifractal Process Based Modeling of Broadband Traffic Processes : Variable Bit Rate Video Traffic
}

\author{
Murali Krishna, Vikram Gadre, Uday Desai \\ SPANN Lab, Dept. of EE, IIT - Bombay \\ Bombay - 400076, INDIA \\ mkrishna@ee.iitb.ac.in
}

\begin{abstract}
We have developed a new approach to model broadband network traffic based on multiplicative multifractal models. In this paper we propose a VVGM (Variable Variance Gaussian Multiplier) multiplicative multifractal model for modeling VBR traffic. The results of the detailed statistical and performance analysis on the original and the synthesized traces are presented in this paper. The results of the proposed model on comparison with the traditionally used fARIMA and the recently introduced Wavelet based video traffic process are shown to be significantly better.

Keywords-Multifractals, long range dependence, VBR traffic
\end{abstract}

\section{INTRODUCTION}

$\mathrm{M}$ ODELING of VBR video traffic has generated a lot of interest in the recent years and several models can be found in the current traffic modeling literature which include Markov models [1], AR based models [2], wavelet models [4], self similar models [3]. Multifractal analysis of video traffic was first done by Mannersalo [5]. Their study was of empirical nature and no model was proposed to synthesize the data traces. The main contribution of this paper is that we are proposing a new model called the Variable Variance Gaussian Multiplier (VVGM) model for the VBR traffic based on the theory of multiplicative multifractal processes. The comparison study between the synthesized traces and the original data are performed to evaluate the extent of similarity between the two. We have also compared the system performance with the more commonly used fARIMA model suggested by Garret [3] and wavelet based models proposed by Ma [4]

\section{Summary of Results and Overview}

We evaluated the multifractal spectrum of the synthesized data and compared it with that obtained for the original traces. The results of the multifractal analysis have shown that the VVGM multiplicative multifractal process possesses the same scaling nature as the original VBR traces. For the analysis of system performance, we studied the queue length distribution of a multiplexed trace driven single server queue. We also analyzed the variance of cell delay and the cell loss as well as the effective bandwidth between the original and the VVGM synthesized traces. The results obtained from these experiments have shown that the traces synthesized by the proposed VVGM multiplicative cascade method matches the original VBR traces in performance related aspects also. The rest of the paper is organized as follows. Section II describes the basic theory of Multiplicative Multifractals while Section III describes the modeling and synthesis of the data. Section IV gives the details of results obtained on multifractal analysis of the original and synthesized data while Section V gives the result of comparison of the Index of Dispersion of counts (IDC). In Section VI, we present the results of the performance analysis of a queueing system using the original and synthesized traces. Section VII compares the effective bandwidth between the original and the synthesized traces and Section VIII concludes the paper with a discussion on the results obtained and suggestions on future work.

\section{Multiplicative Multifractal Processes}

The multiplicative cascade model was introduced by Frisch and Parisi in the context of turbulence analysis in the late 1980s [6]. A cascade is a process that fragments a given set into smaller and smaller sections according to a geometric rule and at the same time divides the measure of the set according to another random rule [7]. The limiting process generated by the cascade describes the way in which the cascade redistributes the mass of the initial set. The multiplicative cascades are shown to possess a multifractal nature [7].

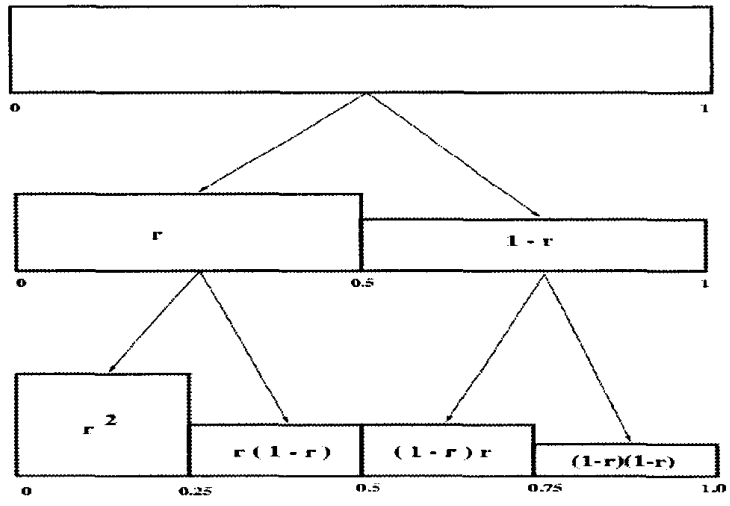

Fig. 1. Multiplicative Cascade Process Construction

Figure 1 illustrates the first steps in the creation of a cascade. Consider an initial set $I_{0}$ denoted by the unit interval $[0,1]$ so that $\mu\left(I_{0}\right)=1$. This measure is divided into two sets $I_{0.0}$ from $[0,1 / 2]$ and $I_{0.1}$ from $[1 / 2,1]$ by multiplying with $r(r<1)$ to the first part and $(1-r)$ to the second part respectively such that the initial measure is preserved. i.e., $\mu\left(I_{0.0}\right)+\mu\left(I_{0.1}\right)=1$. At the next stage, this process is iterated on both the sets $I_{0.0}$ and $I_{0.1}$ to get four sets $I_{0.00}, I_{0.01}, I_{0.10}, I_{0.11}$ such that the measure is again preserved to 1 . This process is iterated for $k$ levels and 
at each stage it can be seen that the total measure is preserved at each stage. The multiplier value with which the measure is multiplied determines the distribution of the measure.

\section{VVGM Multifractal Process}

In the present study, we are proposing a new approach to model cascade processes in which the multiplier values are samples of a Gaussian distribution $f_{R_{i}}(r)$ at each stage $i$. We are modeling the multipliers as normally distributed with mean 0.5 and variances that change at each stage of the cascade generation. The name VVGM (Variable Variance Gaussian Multiplier) multiplicative multifractals is used to denote the process obtained. The synthesis of multiplicative cascades with multiplier distributions varying at each stage of the cascade had shown to yield better approximations to the statistical properties of the generated process than the case where they are invariant with stage [8]. The model is simple to analyze and the synthesis of data based on this model is very fast.

TABLE I

Statistics of THE VBR TRACES: Frame LEVEL

\begin{tabular}{|l|c|c|c|}
\hline \multirow{2}{*}{ Traces } & \multicolumn{3}{|c|}{ Bits / Frame } \\
\cline { 2 - 4 } & $\mu$ (Mean) & $\sigma($ S.D) & Burst factor \\
\hline Starwars & 15598 & 18165 & 11.877 \\
\hline ET & 6459 & 9134.7 & 7.699 \\
\hline MrBean & 16842 & 200014 & 13.6 \\
\hline CrocDundee & 9492 & 9927.6 & 5.4026 \\
\hline
\end{tabular}

The VBR traces used for simulation corresponds to medium to very bursty traces whose Hurst parameter changed from 0.78 to 0.94 . The results of the analysis has shown that the VVGM multifractal model captures the properties of the VBR traffic in all aspects including statistical, scaling and queueing performance. The statistical properties of the VBR traces used for the experiment are given in Table 1.

\section{Multifractal Spectrum}

Multifractality of a process is associated with irregular behavior of it's scaling property. The point wise regularity of such processes keep on changing. Let $X(t)$ be a function and let $P\left(t-t_{0}\right)$ be the polynomial approximation to $X(t)$ at $t=t_{0}$. The function is said to be Holder $\alpha$ at $t_{0}$ if $\exists$ a constant $C$ and a positive real number $\alpha$ such that

$$
\left|X(t)-P\left(t-t_{0}\right)\right| \leq C\left|t-t_{0}\right|^{\alpha}
$$

In the case of multifractal functions, the value of $\alpha$ changes from point to point. An example of such a process is given in Figure 2 which illustrates a VBR trace.

Such a process can be characterized by the multifractal spectrum or the $f(\alpha)$ curve. The $f(\alpha)$ curve gives the fractal dimension of the set of points in the process whose Holder exponent is $\alpha$. Typical $f(\alpha)$ curves are illustrated in Figure 5 to Figure 8. The burstiness of the process can be parametrized based on the nature of the $f(\alpha)$ curve. The region of the curve for $\alpha<1$ indicates very high burstiness in the data [9].

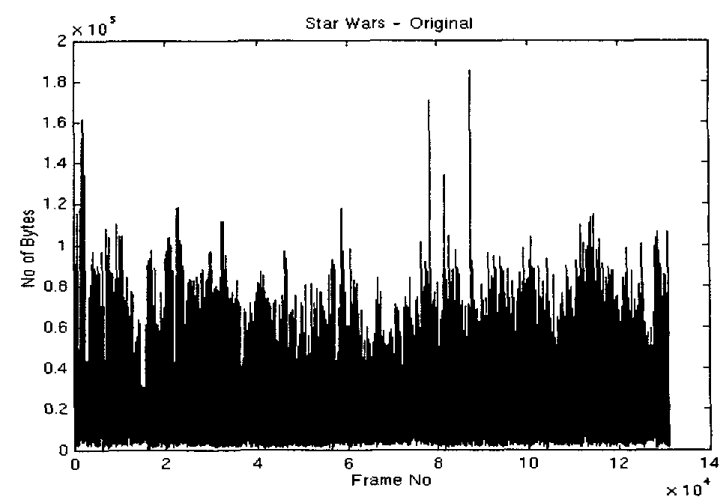

Fig. 2. VBR video trace

\section{Multifractal Modeling Of VBR Traffic}

The empirical observation of VBR video traffic has revealed that it is highly correlated in nature [10]. It is also observed that the degree of burstiness in the VBR video traffic is varying in nature. A realistic model for the VBR traces must also take this factor into account in addition to the long range dependence characteristics. The main factors considered for modeling are (i) data must always be positive, and (ii) the self similarity varies with time. The first assumption is validated from the very nature of data. The second assumption is now a relaxation from the self similar models which requires the same Hurst parameter throughout the process.

\section{Estimating Model Parameters}

For developing the model, the multiplier distribution $f_{R_{i}}(r)$ at each stage $i$ of the cascade construction is to be estimated. We assume $f_{R_{i}}(r)$ is centered around $r=0.5$ so that both $r$ and $(1-r)$ have the same probability distribution. Let $x_{i}^{N},\left(i=1, \ldots, 2^{N}\right)$ denote the process obtained as a result of the cascade construction at stage $N$. Every point in the sequence $x_{i}^{N}$ can be written as the product of several random variables $U_{1} U_{2} \ldots U_{N}$, where $U_{j},(j=1, \ldots, N)$ is either $|r|$ or $|(1-r)|$ which is the multiplier at stage $j$. The algorithm to estimate the multiplier distribution is as follows:

1. Given the data at stage $N, x_{i}^{N}\left(i=1, \ldots, 2^{N}\right)$ (with time resolution of $2^{-N}$ ), the data at stage $N-1$ is obtained by aggregating the consecutive values at stage $N$ over non overlapping blocks of size two. Similarly, given the data at a coarser scale $N-j, x_{i}^{N-j}\left(i=1, \ldots, 2^{N-j}\right)$, we obtain the data at stage $N-(j+1)$ (lesser resolution) by adding consecutive values at stage $j$ over non overlapping blocks of size two, i.e.,

$$
x_{i}^{N-j-1}=x_{2 i-1}^{N-j}+x_{2 i}^{N-j}
$$

for $i=1, \ldots, 2^{N-j}$.

2. An estimate of the multipliers for proceeding from stage $j$ to stage $j+1$ can be obtained as follows:

$$
r_{i}^{(j)}=\frac{x_{i}^{N-j}}{x_{2 i-1}^{N-j-1}}
$$

for $i=1, \ldots, 2^{N-j-1}$. 


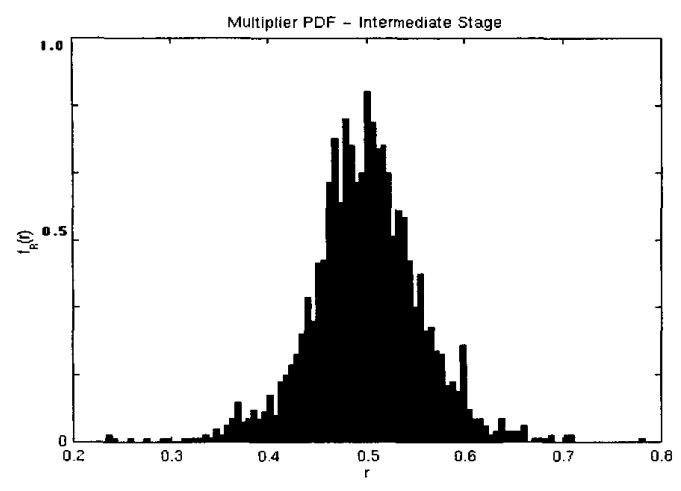

Fig. 3. Histogram of Multipliers

3. We view $\left\{r_{i}^{(j)}, i=1, \ldots, 2^{N-j-1}\right\}$ as samples of the multiplier distribution $f_{R_{j}}(r)$ at scale $j$. Now $f_{R_{j}}(r)$ can be approximated by the histogram of $r_{i}(j)$

4. The variance of the multipliers $\sigma_{j}^{2}$ at each stage $j$ is estimated from the histogram obtained by the previous step. Figure 3 illustrates the multiplier histogram obtained at an intermediate stage in the estimation process.

The process is repeated till the aggregates converge to a single point which can be looked upon as the starting value. The variances at each stage obtained for the traces are plotted in Figure 4. The similarity in the change of multiplier variance with scale for the different traces indicates that the assumption of a multiplicative cascade process for modeling the VBR traces stands valid.

\section{Synthesis of VVGM Process}

For the synthesis purpose, we start from the coarsest value of the aggregate and multiply it at each stage using multiplier values chosen from Gaussian distributions with variances that we estimated in the estimation phase. The algorithm for synthesis of the traces is as follows:

1. Begin with starting value of the aggregate obtained during the estimation phase.

2. At stage $i$, generate random numbers from $N\left(0.5, \sigma_{i}^{2}\right)$ where $\sigma_{i}^{2}$ is the variance at stage $i$.

3. Multiply the starting aggregate value by multipliers generated at each stage from the distributions mentioned earlier to obtain the multiplicative cascade.

\section{Results Of Multifractal ANAlysis}

In order to compare the scaling nature of the original and the simulated traces, we compared the multifractal spectrum or the $f(\alpha)$ curve. We have used $2^{17}$ frames for our experiments which correspond to roughly 1 hour 30 minutes of video. We used the method of moments to obtain the multifractal spectrum of the original and the synthesized traces [9]. Given the data $x_{i}^{N}$, the partition function is defined as

$$
\chi_{m}(q):=\sum_{k=1}^{N / m}\left(\overline{x_{k}^{(m)}}\right)^{q} ; q \in \mathbb{R}
$$

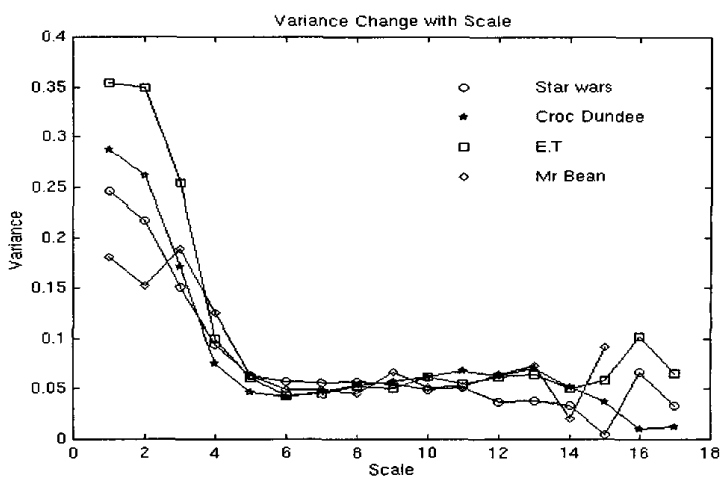

Fig. 4. Variance change with scale

where

$$
\overline{x_{k}^{(m)}}:=\sum_{i=1}^{m} x_{(k-1) m+i}^{m}
$$

The partition function exhibits a scaling nature of the form

$$
\chi_{m}(q)=m^{\tau(q)}
$$

Taking logarithm of (6) gives

$$
\log \chi_{m}(q)=\tau(q) \log m+K
$$

where $K$ is a constant. The parameter $\tau(q)$ is the slope of the $\log$ - log regression curve. In computing the multifractal spectrum, we obtain the values of $\tau(q)$ for different $q$ by performing a least square fitting between $\log \chi_{m}(q)$ against $\log m$. The multifractal spectrum $f(\alpha)$ of the process $x(t)$ can be obtained by finding the Legendre Transform of $\tau(q)$ defined in [9]

$$
f(\alpha)={ }_{q}^{\min }\{q \alpha-\tau(q)\}
$$

The original and synthesized traces showed excellent multifractal scaling of the partition function $\chi_{m}(q)$ for $q \in[-4.5,4.1]$ when taking $m=2,4, \ldots, 2^{17}$. The multifractal spectrum of the original and synthesized traces is compared in Figures 5 and 7. One can see the excellent agreement between $f(\alpha)$ for the original and the $f(\alpha)$ for the VVGM synthesized traces. On the other hand the $f(\alpha)$ spectrum of both the wavelet model and fARIMA depicted in Figures 6 and 8 are concentrated around a single point which clearly depicts that these processes are mono fractal in nature. This clearly has vindicated the superiority of multifractal models for modeling VBR traffic data. Table 2 gives the values of the $\alpha_{\min }$ and $\alpha_{\max }$ obtained on multifractal analysis of the original and the VVGM synthesized VBR traces.

\section{INDEX OF DISPERSION OF COUNTS}

A measure of the burstiness present in the trace can be obtained by obtaining the index of dispersion of counts (IDC) of the process [11]. The IDC of the process at time $t$ is defined as the variance of the number of arrivals in an interval of length $t$ divided by the mean number of arrivals in $t$.

$$
I_{t}=\frac{\operatorname{var}(N(t))}{E(N(t))}
$$




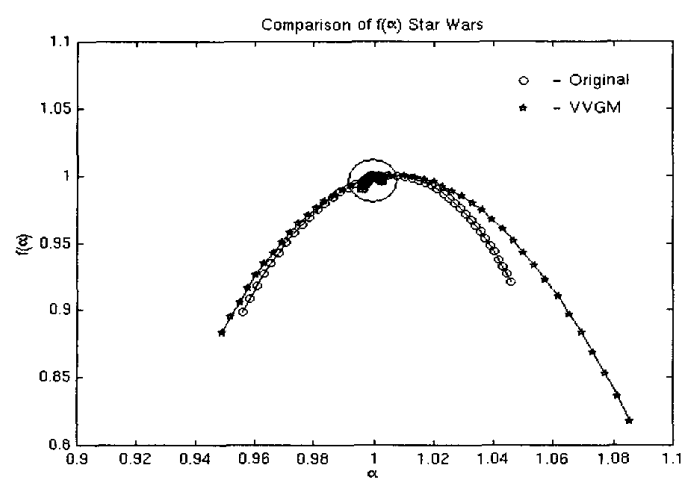

Fig. 5. Comparison of Multifractal Spectrum $f(\alpha)$ - Starwars

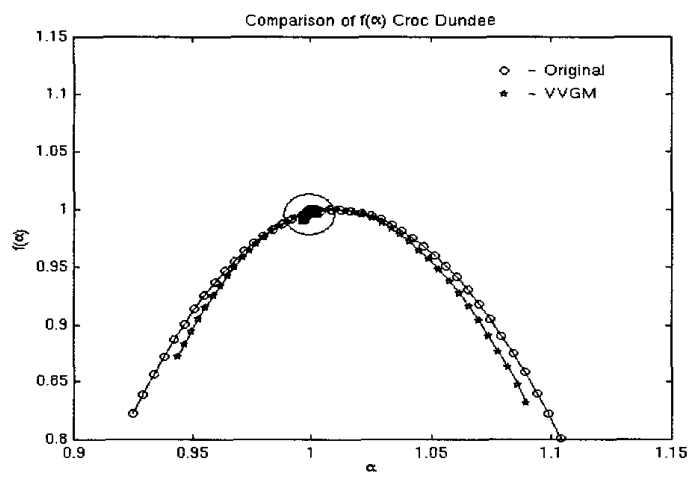

Fig. 6. Comparison of Multifractal Spectrum $f(\alpha)$ - Croc Dundee

where $N(t)$ indicates the number of arrivals in an interval of length $t$. We consider only discrete spaced time instants $\tau_{i}(i>$ $0)$. Denoting the number of arrivals in the $i^{t h}$ interval of duration $T=\tau_{i}-\tau_{i-1}$ by $u_{T}(i)$, we have,

$$
I_{n}(T)=\frac{\operatorname{var}\left(\sum_{i=1}^{n} u_{T}(i)\right)}{E\left(\sum_{i=1}^{n} u_{T}(i)\right)}
$$

The IDC is a function of the length of time $T$, over which the counts sequence is constructed from the original process. The IDC of the original VBR traces and the synthesized traces are compared in the Figures $9-12$.

\section{RESUlts OF QUEUEING Simulations}

For our queueing analysis, we make the following assumptions: The time is discretized into frame durations, i.e the recip-

TABLE II

COMPARISON OF $\alpha$ PARAMETERS

\begin{tabular}{|c|c|c|c|c|}
\hline \multirow{2}{*}{ Traces } & \multicolumn{2}{|c|}{$\alpha_{\min }$} & \multicolumn{2}{c|}{$\alpha_{\max }$} \\
\cline { 2 - 5 } & Original & VVGM & Original & VVGM \\
\hline Starwars & 0.9416 & 0.9458 & 1.0585 & 1.0774 \\
ET & 0.8994 & 0.9287 & 1.0788 & 1.0894 \\
MrBean & 0.9067 & 0.9250 & 1.0654 & 1.0906 \\
CrocDundee & 0.9279 & 0.9444 & 1.1156 & 1.1013 \\
\hline
\end{tabular}

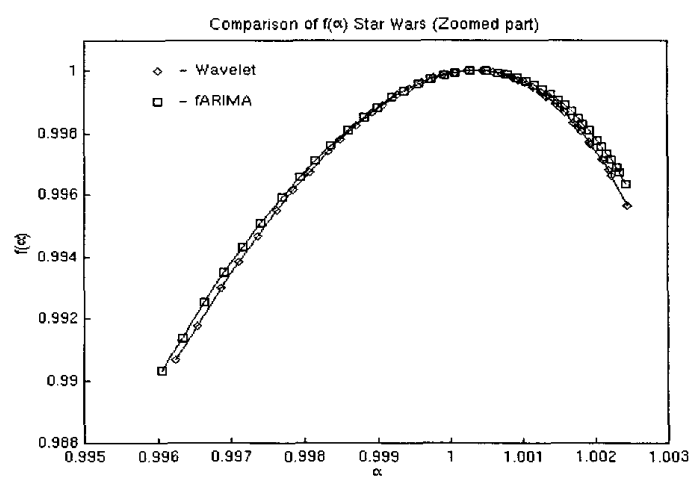

Fig. 7. Comparison of Multifractal Spectrum $f(\alpha)$ - Starwars

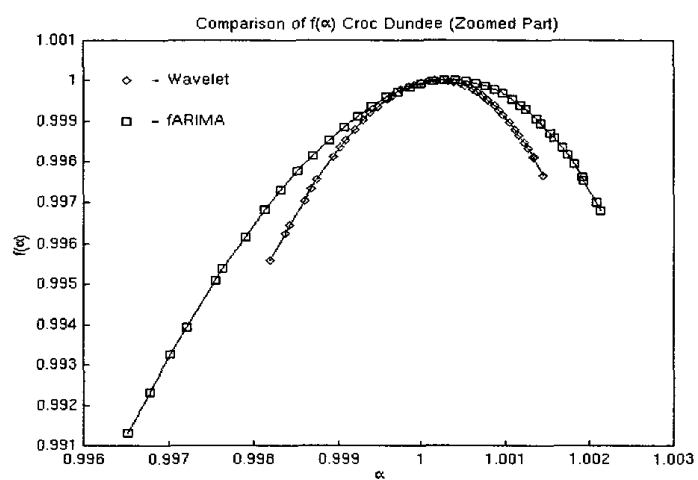

Fig. 8. Comparison of Multifractal Spectrum $f(\alpha)$ - Croc Dundee

rocal of the frame rate $t_{r}=25 \mathrm{~s}^{-1}$. The trace data is discretized into ATM cells carrying 48 bytes of payload. Instead of individual cells, we consider the frame data as a fluid which flows into the buffer at a constant rate. This method is applicable if the cell rate remains the same a time interval which is longer than the cell inter arrival times.

\section{Behavior of Queue Lengths}

In order to study the queue length distribution, we plot the complimentary distribution of the queue length process and observe the decay of the probability that the queue length increases beyond a particular threshold. The link capacity of the queue is fixed at $100 \mathrm{Mbps}$. In order to compare the performance, we also did the simulations using the fARIMA process with $H=0.83$ and wavelet model synthesized traces. Figure 13 gives the logarithm of complementary distribution function of the queue length. It is interesting to note that while the original traces and the vvgm simulated traces gave identical queue length distributions, the fARIMA traces shows marked variation after a particular range of queue length.

\section{Variance of Cell Delay}

Cell delay variation (CDV) or jitter can have a significant impact on the quality of a video stream [12]. In the present study, we have analyzed the cell delay variation as a function of the buffer utilization factor for the original and the simulated traces. The 


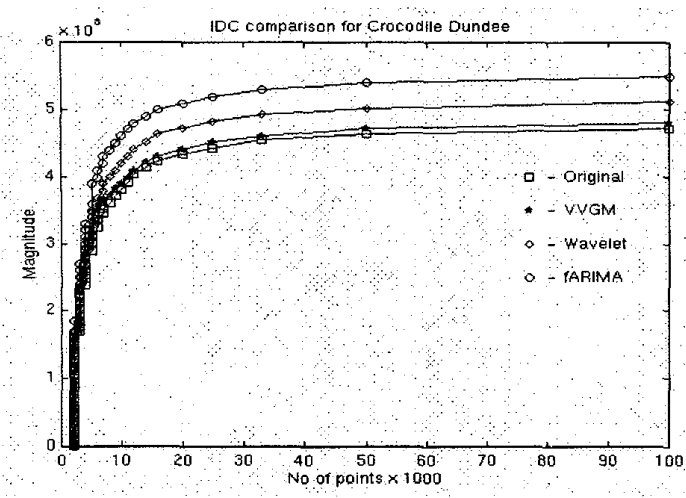

Fig. 9. Comparison of IDC for Croc Dundee

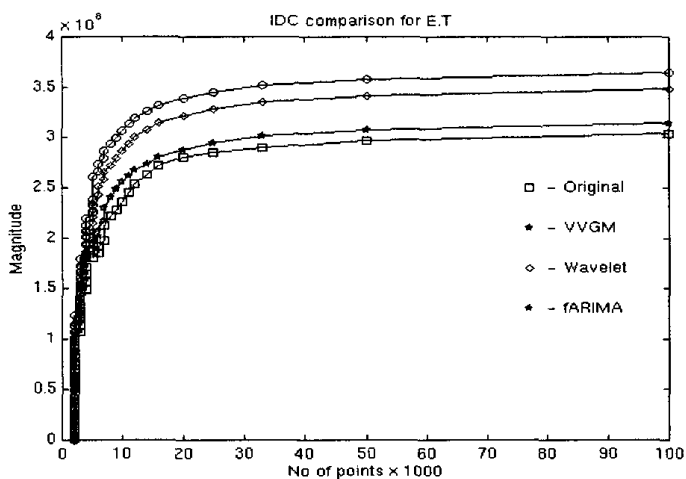

Fig. 10. Comparison of IDC for E.T

offered load is given by $\rho=E[x] / R$ where $E[x]$ is the average cells per frame for the entire sequence and $R$ is the number of cells transmitted per frame time. Figure 14 shows the variation of cell delay variance for the original and the simulated traces.

\section{Comparison of Cell Loss}

In this experiment we have simulated the variation of cell loss with a finite buffer keeping the data link rate of the output a constant. The buffer utilization factor is varied as $\lambda=E[x] / B$ where $E[x]$ is the mean of the trace and $B$ is the buffer length. Figure 15 gives the cell loss probability with $\lambda$. Note that the VVGM multifractal process does a better job of approximating the cell loss probability for $\lambda>0.65$.

\section{COMPARISON OF EFFECTIVE BANDWIDTH}

The concept of an Effective Bandwidth was proposed by Kelly [13] in the context of stochastic models for statistical sharing of resources. The effective bandwidth surface provides both a graphical and quantitative view of the performance implications of broadband traffic sources with respect to statistical resource sharing. This has many applications to issues such as statistical multiplexing, call admission control etc. The effective bandwidth is defined as

$$
B(\theta, T)=\frac{\log E\left[\exp \left(\theta x_{k}^{T}\right)\right]}{\theta T}
$$

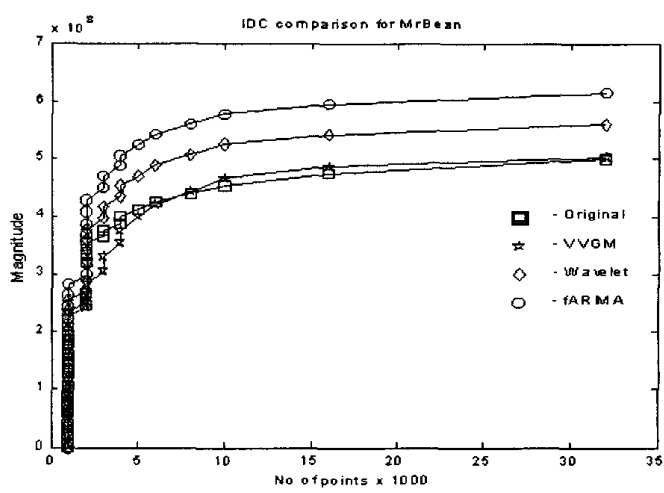

Fig. 11. Comparison of IDC for Mr Bean

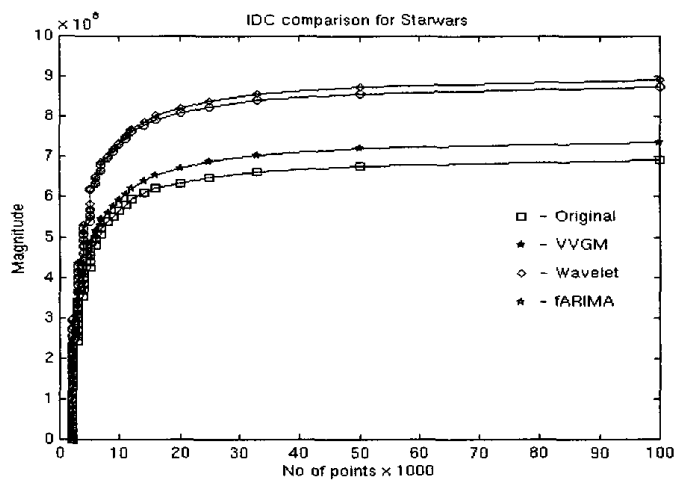

Fig. 12. Comparison of IDC for Star Wars

where $\theta$ is the scale space and $T$ is the time scale. For example, $\theta$ may be measured in bytes ${ }^{-1}$ and $T$ might be measured in seconds. $x_{k}^{T}$ is the workload arriving in the time duration $T$ which is the aggregate of the video trace over the time considered.

$$
x_{k}^{T}=\sum_{\tau=k}^{\tau=k+T} x(\tau)
$$

The two dimensional surface described by $B(\theta, T)$ is evaluated over a range of values for $\theta \in\left\{\ldots, 10^{-5}, \ldots, 10^{-1}\right\}$ and for $T \in\left\{2,4, \ldots, 2^{16}\right\}$ Figure 16 compares the effective bandwidth surface for the original and the various synthesized data traces.

\section{CONCLUSIONS}

In this paper, we have proposed a new model for the synthesis of VBR video traffic using the VVGM multifractal model. We have synthesized the VBR trace data based on the VVGM multiplicative multifractal model for different video traces. We specifically investigated the multifractal spectrum curve of the original and the synthesized traces. The results of the scaling analysis show that the VBR traces possess multifractal nature and the VVGM multifractal process does a very good job of approximating the multifractal property of the VBR traces. In order to further validate the model, we conducted queueing ex- 


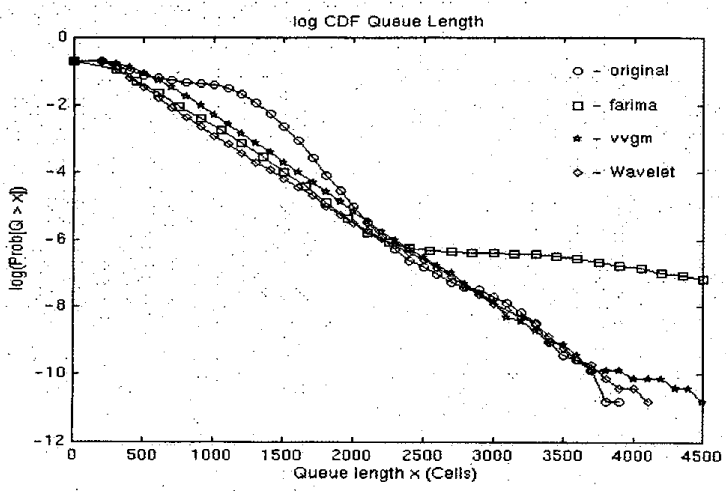

Fig. 13. Complimentary Distribution of Queue Length

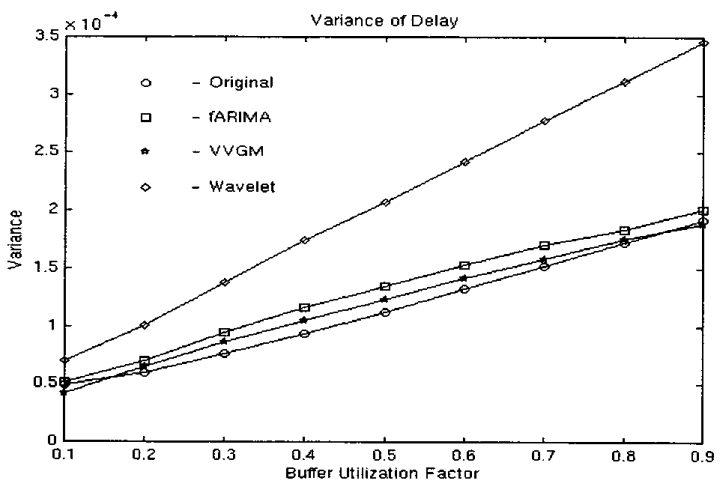

Fig. 14. Cell Delay Variance with Buffer Utilization

periments on the the traces to study the queue length distribution, the cell delay variation and the loss process. The queueing simulation results have shown that the VVGM multifractal process does a better job at modeling VBR traces than the fARIMA or the wavelet based model. The future directions of work include the study of predictive control of network resources based on this model.

\section{ACKNOWLEDGMENTS}

The authors would like to thank the reviewers for their comments and suggestions.

\section{REFERENCES}

[1] D. M. Lucantoni, M. F. Neuts and A. R. Reibman, "Methods for Performance Evaluation of VBR Video Traffic Models", IEEE/ACM Transactions on Nerworking, Vol 2, No 2, pp 176-180, April 1994

[2] A. Adas, "Supporting Real Time VBR Video using Dynamic Reservation based Linear Prediction", Proceedings of IEEE Infocomm '96, pp 14761483, 1996.

[3] M. W. Garrett, W. Willinger, "Analysis, Modeling and Generation of Self Similar VBR Video Traffic", Proceedings of ACM SIGCOMM '94 Conference, pp 269-280, 1994.

[4] S. Ma, C. Ji, "Modeling Video Traffic in the Wavelet Domain", Proceedings of IEEE INFOCOM'98 pp.201-208, March 1998

[5] P. Mannersalo, I. Norros, "Multifractal Analysis of Real ATM Traffic: a First Look", COST 257TD(97), VTT Information Technology. Finland, 1997.

[6] U. Frisch, G. Parisi, "Turbulence and Intermittency", in Turbulence and predictability in Geophysical Fluid Dynamics and Climate Dynamics, pp 84-88, Amsterdam, North Holland, 1985

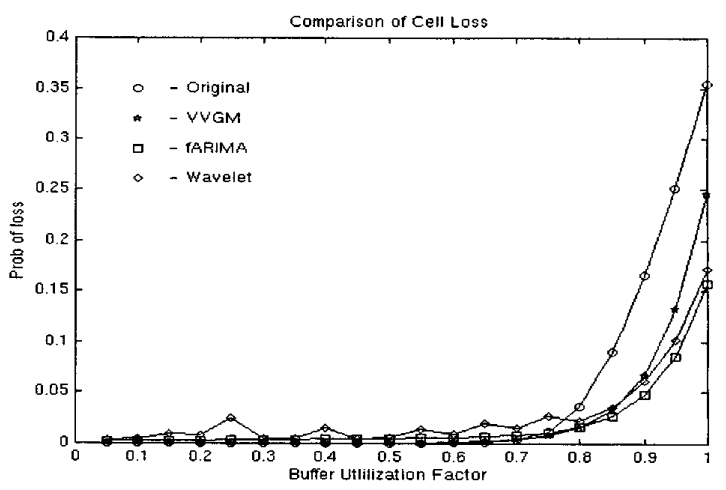

Fig. 15. Probability of Cell Loss with Buffer Utilization
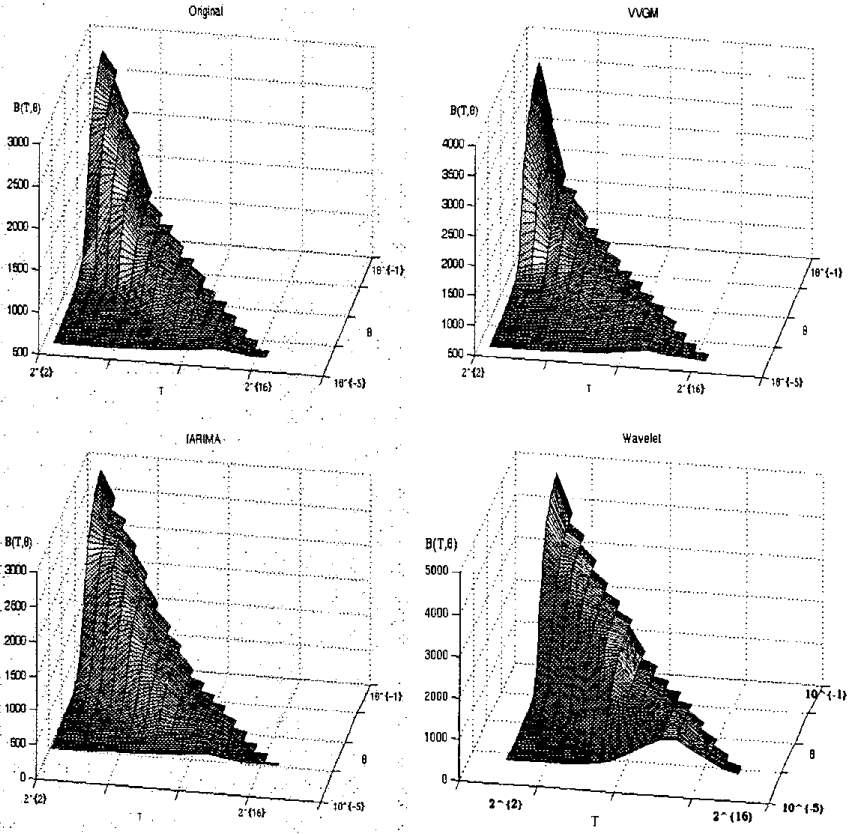

Fig. 16. Comparison of Effective Bandwidth

[7] Richard Holley and Edward. C. Waymire, "Multifractal Dimensions and Scaling Exponents for Strongly Bounded Random Cascades", The Annals of Applied Probability, Vol 2, pp 819-845, April 1992.

[8] Murali Krishna. P, V. M. Gadre and U. B. Desai, "Synthesis of Multifractal Processes Using Variable Variance Gaussian Multiplier Model", Proceedings of NCC-2001, IIT - Kanpur, pp 163-166, January 2001.

[9] Rudolf Riedi, "Introduction to Multifractals", Rice University Technical Report, September 1999.

[10] J. Beran, R. Sherman, M. S. Taqqu and W. Willinger, "Variable Bit Rate Video Traffic and Long Range Dependance", IEEE Transactions on Communications, Vol 43, No 4, pp 1566-1579, April 1995.

[11] R. Gusella, "Characterizing the variability of arrival processes with indexes of dispersion", IEEE Jr. Select. Areas in Communication, Vol 9, No 2, pp 203-211, Feb 1991.

[12] W. Zhu, Y. T. You and Y. Q. Zhang, "End to End Modeling and Simulation of MPEG-2 Transport Streams over ATM Networks with Jitter", IEEE Transactions in Circuits and Systems for Video Technology, Vol 8, No 1, pp 9-12, February 1998.

[13] F. P. Kelly, S. Zachary and I. B. Ziedins, "Notes on effective bandwidths", Stochastic Networks: Theory and Applications, Oxford University Press, pp 141-168, 1996. 